\title{
ELECTROABSORPTION BY IMPURITIES AND DEFECTS IN SEMI-INSULATING CdTe
}

\author{
G. NEU, Y. MARFAING, R. TRIBOULET and M. ESCORNE
}

Laboratoire de Physique des Solides, C. N. R. S.,

1, place A. Briand, 92190 Meudon/Bellevue, France

\begin{abstract}
Résumé. - Des expériences d'électroabsorption extrinsèque permettent de comparer les spectres des défauts dans CdTe compensé non dopé et dopé au chlore de 1,2 à 1,6 eV. Trois groupes de transitions sont relevés :

- des transitions bande de valence-niveaux donneurs semi-profonds $\left(E_{\mathrm{c}}-0,02\right.$ à $\left.0,03 \mathrm{eV}\right)$ liés à la présence du chlore et d'impuretés du groupe III,

- des transitions à partir de niveaux accepteurs peu profonds $\left(E_{0}+0,05 \mathrm{eV}\right)$ vers la bande de conduction,

- une transition de basse énergie $\left(E_{\mathrm{c}}+0,13 \mathrm{eV}\right)$. Cette dernière est étudiée en fonction de l'orientation du cristal et de la polarisation de la lumière afin de préciser la nature du défaut qu'elle implique, et particulièrement sa symétrie.

Abstract. - Electroabsorption experiments have been conducted on semi-insulating CdTe prepared by THM without intentional doping, and with addition of chlorine. Three groups of optical transitions due to localized levels have been observed in the photon energy range from $1.2 \mathrm{eV}$ to $1.6 \mathrm{eV}$ :

- transitions from the valence band to shallow donor levels $\left(E_{\mathrm{c}}-0.02,0.03 \mathrm{eV}\right)$, associated with chlorine or group III donors,

- transitions from shallow acceptors $\left(E_{\mathrm{v}}+0.05 \mathrm{eV}\right)$ to the conduction band,

- transitions from a deep acceptor level $\left(E_{\mathrm{v}}+0.13 \mathrm{eV}\right)$ to a shallow donor level.

The latter level is usually attributed to a donor-cadmium vacancy complex, which prompted us to undertake a more systematic study of the transition. Various electroabsorption spectra were recorded as a function of temperature, crystal orientation and light polarization. Strong dichroic effects were observed, the analysis of which should allow one to determine the symmetry of the complex involved.
\end{abstract}

1. Introduction. - Extrinsic electromodulation experiments have already provided much information on impurity centers in semiconductors [1, 4]. Early work [5, 6] on CdTe has shown the existence of localized levels, which probably imply the presence of native defects. These defects play an important role in the electrical compensation of the material. In the case of a high degree of compensation a study of the centers involved using electroabsorption seems particularly suitable, owing to the high resistivity of the material.

Therefore, we have undertaken spectroscopy of semi-deep, localized levels in semi-insulating samples, using the electric field as a modulation parameter.

Two kinds of samples prepared by the solution growth technique THM were studied : the ones grown at $700^{\circ} \mathrm{C}$ were not intentionally doped, and the other ones, grown at $900^{\circ} \mathrm{C}$ were doped with chlorine to a level of $2 \times 10^{17} \mathrm{~cm}^{-3}$.

1.1 ElECTROABSORPTION SPECTRA. - The spectra recorded at $77 \mathrm{~K}$ on undoped samples (Fig. 1a) display two sets of oscillations : the larger, $D_{1}$, is located near the start of the absorption edge. The signal spreads out around $1.565 \mathrm{eV}$. A second group of oscillations, $A_{1}$, is centered around $1.545 \mathrm{eV}$. The signal amplitudes are proportional to the square of the electric field. At low temperature (Fig. 1b), the shape of $D_{1}$ as well as its quadratic amplitude are not modified. This signal corresponds to an optical transition from the valence band to a donor level located $30 \mathrm{meV}$ below the conduction band at $77 \mathrm{~K}$.

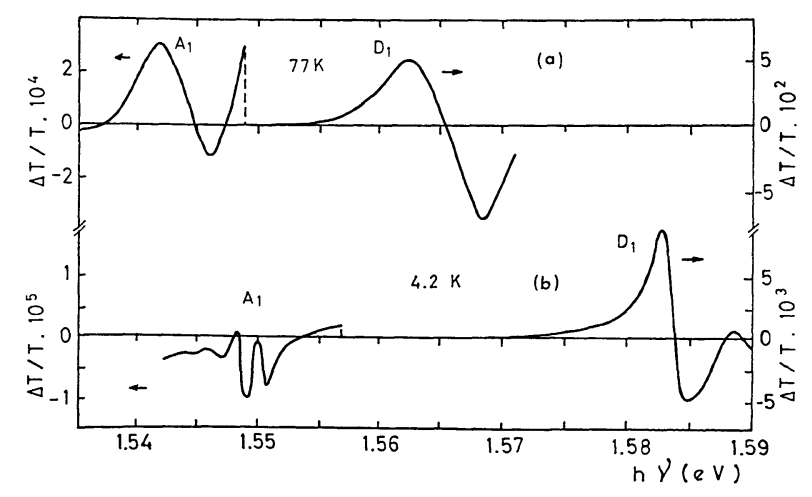

Fig. 1. - Electroabsorption spectra at $77 \mathrm{~K}(a)$ and $4.2 \mathrm{~K}(b)$ for undoped CdTe. a) $E=5000 \mathrm{~V} / \mathrm{cm}, b) E=1000 \mathrm{~V} / \mathrm{cm}$. 
The theoretical analysis of such electroabsorption signals can be rather easily formulated in the case of a valence band to hydrogenic donor level transition [7]. Calculating the optical transition probability between the valence band states perturbed by the field, $F$, and the ground state of the donor, the absorption coefficient, $\alpha$, is obtained as :

$$
\alpha=\alpha_{0}+A(\hbar \Delta \omega) F^{2}
$$

where $\alpha_{0}$ is the absorption coefficient at zero field as given for example by Dumke [8], and $A(\hbar \Delta \breve{\omega})$ is a function of the incident light energy. This analysis allows us to explain the quadratic variation of the amplitude with electric field.

At $4.2 \mathrm{~K}$ the set $A_{1}$ consists of a series of narrow oscillations around $1.55 \mathrm{eV}$. This signal can be assigned to a transition from acceptor levels localized at about $55 \mathrm{meV}$ above the valence band to the conduction band. The displacement of $A_{1}$ with temperature is normal. However, $D_{1}$, taking as reference the energy at which the signal equals zero, increases by $19 \mathrm{meV}$ when the temperature is reduced from $77 \mathrm{~K}$ to $4.2 \mathrm{~K}$, whereas the bandgap energy only increases by $11 \mathrm{meV}$.

This behavior indicates an anomalous variation of donor ionization energy as a function of temperature. This variation was previously observed in photocapacitance [9] and piezoreflectance experiments [10]. This level also seems to be the origin of the luminescence line observed at $1.584 \mathrm{eV}$ at $4.2 \mathrm{~K}$ in undoped or indium doped material. Since in undoped material the residual donors are postulated to be of group III, the donor center $D_{1}$ can be associated with group III impurities occupying non substitutional sites or complexed with native defects.

The electroabsorption spectrum in the same range of energy is modified by the presence of chlo-

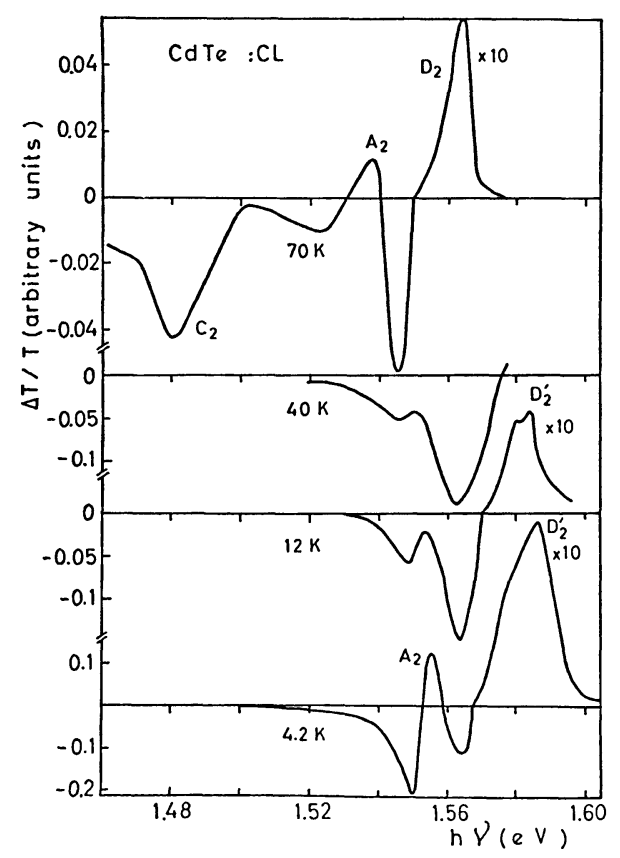

FIG. 2. - Dependence of the $E$. $A$. spectra on temperature for chlorine doped CdTe $(E=1000 \mathrm{~V} / \mathrm{cm})$. rine (Fig. 2). At $70 \mathrm{~K}$, with unpolarized light, the high energy region displays two peaks which increase as the square of the field. The peak $D_{2}$, located around $1.563 \mathrm{eV}$ is preceeded by a weaker peak $A_{2}$ at $1.54 \mathrm{eV}$. The low energy region shows a wide negative peak $C_{2}$ around $1.48 \mathrm{eV}$, which increases linearly with the field. Thus besides donor-type and acceptor-type transitions, a new transition $\mathrm{C}_{2}$ has appeared.

Figure 2 also shows the evolution of the spectrum at low field with decreasing temperature ; two salient features are observed :

(1) The $C_{2}$ peak which exists at $70 \mathrm{~K}$ disappeared at $40 \mathrm{~K}$.

(ii) A new high energy peak $D_{2}$ appears at low temperature, at the same energy as $D_{1}$ in undoped samples.

In compensated chlorine doped material a donor level at $28 \mathrm{meV}$ below the conduction band has already been determined by a time of flight technique [11]. This level can be associated with transition $D_{2}$ observed at $1.563 \mathrm{eV}$ at $77 \mathrm{~K}$. The shift of this level with decreasing temperature, which can be easily observed with high field spectra, corresponds to the bandgap temperature dependence. The $D_{2}$ transition can then be related to the presence of a residual group III impurity, similar to $D_{1}$.

Since the shift of the peak $C_{2}$ is proportional to the field, the zero field transition energy can be found by extrapolation to be $1.463 \mathrm{eV}$. The ground state level of this transition is currently attributed to an acceptor complex which consists of a cadmium vacancy and a chlorine atom on the nearest tellurium site. The final state is thought to be a shallow donor state [12]. This complex center plays a dominant role in self compensating donors and deserves more detailed study.

1.2 LINEAR DICHROISM EFFECTS. - The electric field induces dichroism in the absorption which can be used to get information about the symmetry o: complexes. Figure 3 is an example of such behavior When the electric field is applied along the ( $\overline{1} 10$ direction, and the incident light is coming alon the (111), the sign of the $C_{2}$ peak is opposite, depeni

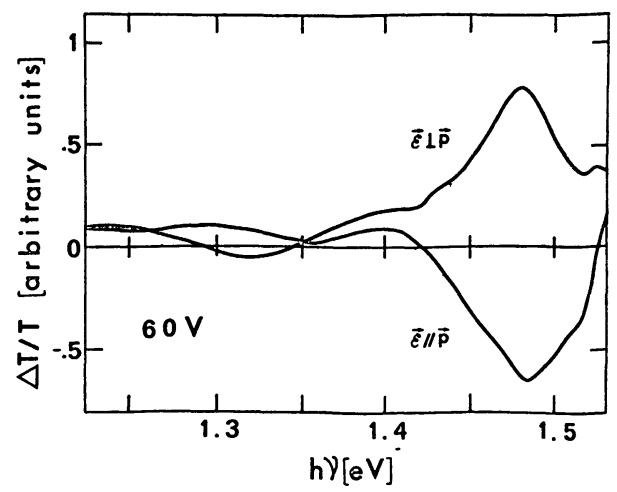

FIG. 3. - Linear dichroism induced by the electric field peak $(E=400 \mathrm{~V} / \mathrm{cm})$ 
ing on whether the light is polarized parallel or perpendicular to the electric field. Figure 4 shows that when the field is increased, a new narrow oscillation emerges from the broad $C_{2}$ peak.

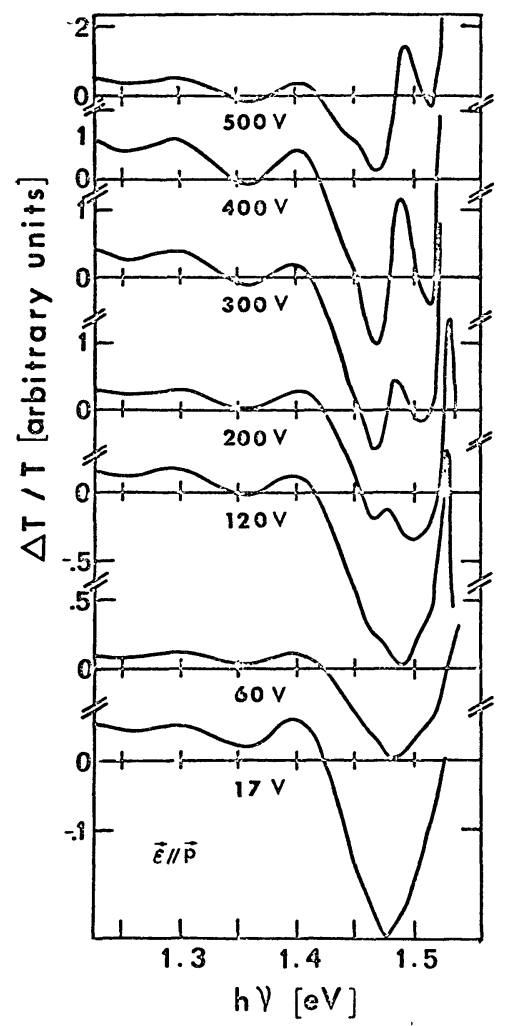

FIG. 4. - Dependence of the E. A. spectrum $(E / / P)$ on increasing electric fields (Sample thickness $=1.5 \mathrm{~mm}$ ).

Let us assume that the acceptor complexes are oriented along (111)'s as is expected for a group VII donor-cadmium vacancy association. In our sample configuration only two sets of equivalent dipoles have an electric moment modulated by the field, if the electric dipole is taken to exist along the defect axis.

To a first approximation, absorption of light is proportional to the square of the scalar product between the polarization vector $\mathbf{P}$ and the electric moment D :

$$
\alpha \simeq(\mathbf{P} \times \mathbf{D})^{2}
$$

The expression for the electroabsorption can be decomposed into two parts :

$$
\Delta \alpha \simeq \sum(\mathbf{P} \times \mathbf{D})(\mathbf{P} \times \Delta \mathbf{D})+(\mathbf{P} \times \Delta \mathbf{D})^{2} .
$$

Because of the existence of an equal number of defect dipoles oriented in opposite directions, the first term disappears upon summation. If we assume that the perturbation of electric moment is of the form :

$$
\Delta \mathbf{D}=A \mathbf{D}
$$

i. e., parallel to the electric moment, the second part of $\Delta \alpha$ is proportional to the square of the cosine of the angle between the polarization vector and the electric moment of the dipoles.

This gives the theoretical variation of $\Delta \alpha$ with the polarization angle $\delta$ referred to the field direction shown by the dotted line in figure 5 . The experimental signal shows a quite different behavior. The amplitude at the maximum is phase shifted with respect to the theoretical curve, and there is a sign inversion during half of the period. To come to more definite conclusions, this type of experiment has to be made on crystals of different orientations. However, it seems that the symmetry of the complex $C_{2}$ could be less simple than usually considered. On the other hand, electric birefringence effects could play an important role as demonstrated in $\mathrm{CuCl}$ by Daunois and Deiss [13].

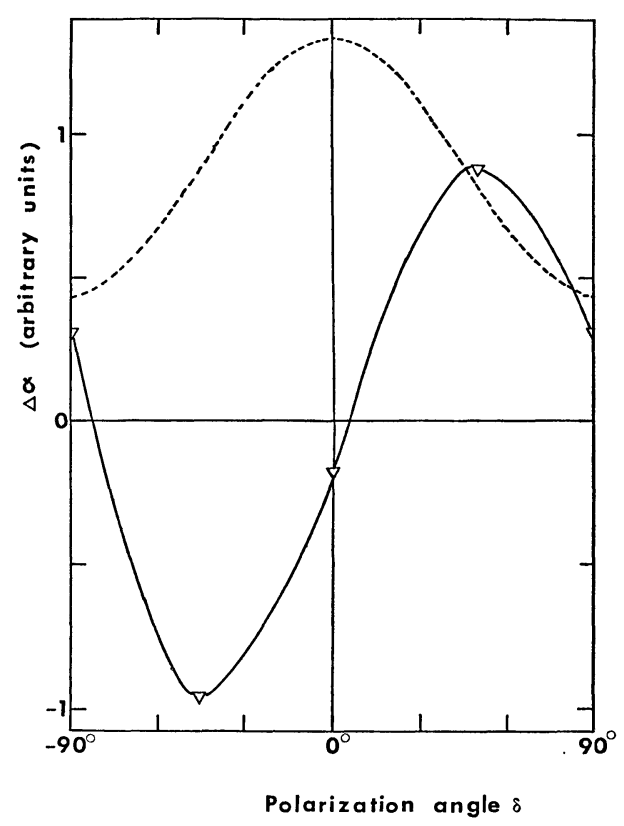

Fig. 5. - Comparison between theoretical variation (dotted line) and experimental measurements (solid line) of E. A. signal with respect to polarization orientation (referred to electric field direction).

2. Conclusion. - The results presented in this paper show that differential electroabsorption is a sensitive technique to detect localized levels in high resistivity semiconductors or insulators.

For chlorine compensated CdTe, electroabsorption spectra display transitions associated with the electronic levels which dominate the self compensation process : a donor level at $E_{\mathrm{c}}-28 \mathrm{meV}$ and an acceptor level at $E_{\mathrm{v}}+130 \mathrm{meV}$.

Transitions originating from the last level exhibit a strong linear dichroism effect which cannot be simply interpreted with the usal assumption of (111) oriented acceptor complexes. 


\section{References}

[1] Jonath, A. D., Voronkov, E. and Bube, R. H., J. Appl. Phys. 46 (1975) 1754.

[2] Ivanov, V. S., Stopachinsky, V. B. and Chapnin, V. A., Sov. Phys. Semicond. 5 (1971) 83.

[3] Vul, B. M., Vavilov, V. S., Ivanov, I. S., Stopachinsky, V. B. and Chapnin, Y. A., Int. Symposium on CdTe, Strasbourg (1971).

[4] BAuer, K. S., J. Elect. Mat. 4 (1975) 1067.

[5] Lorenz, M. R., Segall, B. and Woodbury, H. H., Phys. Rev. 134 (1964) A 751

[6] Agrinskaya, N. V., Arkad'eva, E. N., Matveev, O. A. and Rud, Uy. V., Sov. Phys. Semicond. 2 (1969) 776.

[7] To be published.
[8] Dumke, W. P., Phys. Rev. 132 (1963) 1998.

[9] Marfaing, Y. and Triboulet, R., Int. Symposium on CdTe, Strasbourg (1971).

[10] Camassel, J., Auvergne, D., Mathieu, H., Triboulet, R. and Marfaing, Y., Solid State Commun. 13 (1973) 63.

[11] Canali, C., Ottaviani, G., Bell, R. O. and Wald, F. V., J. Phys. Chem. Solids 35 (1974) 1405.

[12] Agrinskaya, N. V., Arkad'eva, E. N. and Matveev, O. A., Sov. Phys. Semicond. 5 (1971) 767.

[13] Daunois, A. and Deiss, J. D., Colloque sur les propriétés optiques des semiconducteurs à grande bande interdite. 1973, J. Physique Colloq. 35 (1974) C3-53. 